\title{
DIPOLE RESONANCE SPLITTING AND SHELL STRUCTURE PECULIARITIES OF ${ }^{52} \mathrm{Cr}$ NUCLEUS
}

\author{
Goncharova N.G., Dolgodvorov A.P. \\ Lomonosov Moscow State University Skobeltsyn Institute of Nuclear Physics, Russia \\ E-mail: n.g.goncharova@gmail.com
}

Microscopic description of giant resonances and resonance of higher multipolaties (MGR) in ${ }^{52} \mathrm{Cr}$ and ${ }^{54} \mathrm{Fe}$ nuclei was performed in the multiparticle shell model (MSM). The resonances in nuclear excitations cross sections according to MSM are results of doorway-states' collectivisation. This approach was quite successful in interpretations of the main peaks locations on the energy axis for magic nuclei but not for the even-even non-magic ones. Some advance in the interpretation of MGR fragmentation could be attained in the ParticleCore Coupling Version of MSM (PCC SM). PCC SM takes into account the spreading of hole configurations among the states $(A-1)$ daughter nuclei [1]. A full set of basic configurations generating $E 1$ resonances could be obtained from the hole state distribution's analysis revealed in pick-up reaction spectroscopy. This method allows to get a realistic description of $E 1$ resonance in ${ }^{54} \mathrm{Fe}$ nucleus [2]. The ${ }^{54} \mathrm{Fe}$ nucleus has two additional protons in comparison with ${ }^{52} \mathrm{Cr}$ in unfilled $1 f_{7 / 2}$ subshell. The spectroscopic factors' distributions in both nuclei show considerable fragmentation of $1 f_{7 / 2}$ subshell as well as deeper subshells. The energy splitting of subshells revealed in the pick-up reaction spectroscopy of ${ }^{52} \mathrm{Cr}$ together with isospin splitting are the main sources of strengths of $E 1$ resonance fragmentation. The results of PCC SM calculations based on spectroscopy [3] and the comparison with experiment [4] are shown in the Fig.1.

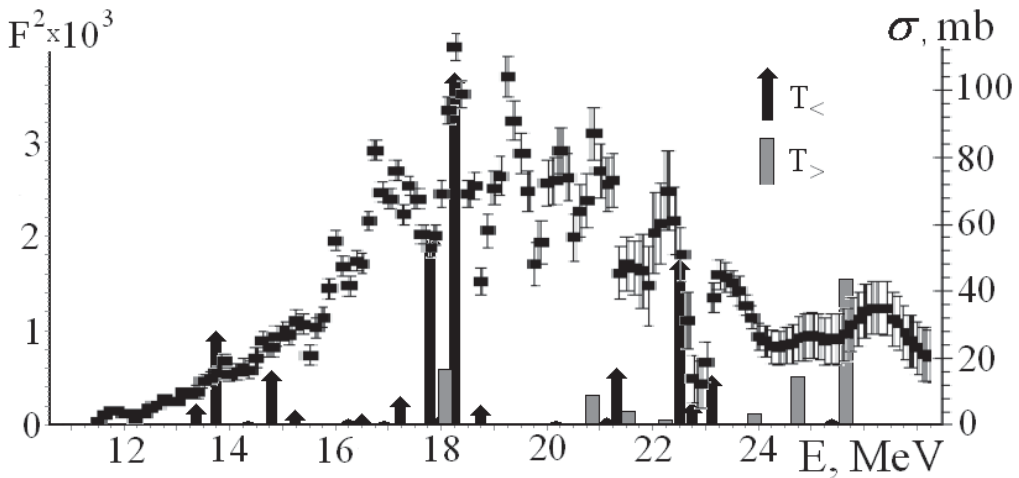

Fig. 1. E1 resonance in ${ }^{52} \mathrm{Cr}$ nucleus.

1. N.G.Goncharova, N.P.Yudin // Phys. Lett. B. 1969. V.29. P.272.

2. N.G.Goncharova, A.P.Dolgodvorov// Phys. of At. Nucl. 2014. V.77. P.200.

3. S.Fortier, E.Hourani, M.N.Rao, et al. // Nucl. Phys. A. 1978. V.311. P.324.

4. B.I.Goryachev, B.S.Ishkhanov, I.M.Kapitonov, et al. // Izv.AN SSSR. S. Fiz. 1969. V.33. P.1736. 